\section{Fibrinogen gamma gene rs2066865 and risk of cancer-related venous thromboembolism}

\author{
Benedikte Paulsen, ${ }^{1}$ Hanne Skille, ${ }^{1}$ Erin N. Smith, ${ }^{2}$ Kristian Hveem $, 3,4,5$ \\ Maiken E. Gabrielsen, ${ }^{4,5}$ Sigrid K. Brækkan, ${ }^{1,6}$ Frits R. Rosendaal, ${ }^{1,7}$ \\ Kelly A. Frazer, ${ }^{1,2}$ Olga V. Gran ${ }^{1}$ and John-Bjarne Hansen ${ }^{1,6}$
}

${ }^{1}$ K.G. Jebsen Thrombosis Research and Expertise Center (TREC), Department of Clinical Medicine, UiT - The Arctic University of Norway, Tromsø, Norway; ${ }^{2}$ Department of Pediatrics and Rady's Children's Hospital, University of California, San Diego, La Jolla, CA, USA; ${ }^{3}$ St. Olavs Hospital, Trondheim University Hospital, Trondheim, Norway; ${ }^{4}$ HUNT Research Centre, Department of Public Health and General Practice, Norwegian University of Science and Technology, Levanger, Norway; ${ }^{5}$ K.G. Jebsen Center for Genetic Epidemiology, Department of Public Health, Norwegian University of Science and Technology, Trondheim, Norway; ${ }^{6}$ Division of Internal Medicine, University Hospital of North Norway, Troms $\varnothing$, Norway and ${ }^{7}$ Department of Clinical Epidemiology, Leiden University Medical Center, Leiden, the Netherlands

\section{ABSTRACT}

$\mathrm{V}$ Tenous thromboembolism (VTE) is a frequent complication in patients with cancer. Homozygous carriers of the fibrinogen gamma gene (FGG) rs2066865 have a moderately increased risk of VTE, but the effect of the FGG variant in cancer is unknown. We aimed to investigate the effect of the FGG variant and active cancer on the risk of VTE. Cases with incident VTE $(n=640)$ and a randomly selected age-weighted sub-cohort $(n=3,734)$ were derived from a population-based cohort (the Tromsø study). Cox-regression was used to estimate hazard ratios (HR) with $95 \%$ confidence intervals (CI) for VTE according to categories of cancer and FGG. In those without cancer, homozygosity at the FGG variant was associated with a $70 \%$ (HR 1.7, 95\% CI: 1.2-2.3) increased risk of VTE compared to non-carriers. Cancer patients homozygous for the FGG variant had a twofold (HR 2.0, 95\% CI: 1.1-3.6) higher risk of VTE than cancer patients without the variant. Moreover, the six-months cumulative incidence of VTE among cancer patients was $6.4 \%$ (95\% CI: 3.5-11.6) in homozygous carriers of FGG and 3.1\% (95\% CI: 2.3-4.7) in those without risk alleles. A synergistic effect was observed between rs 2066865 and active cancer on the risk of VTE (synergy index: 1.81, 95\% CI: 1.02-3.21, attributable proportion: 0.43 , $95 \%$ CI: 0.11-0.74). In conclusion, homozygosity at the FGG variant and active cancer yielded a synergistic effect on the risk of VTE.

\section{Introduction}

Venous thromboembolism (VTE), a collective term for deep vein thrombosis (DVT) and pulmonary embolism (PE), is a common disease associated with substantial short- and long-term morbidity and mortality. ${ }^{1,2}$ The incidence of VTE is 1-2 in 1,000 people/ year, and it increases steeply with age. ${ }^{3}$ Malignant disease is associated with a four- to seven-fold increased risk of VTE, and 20-25\% of all first lifetime VTE-events are cancer-related. ${ }^{4,5}$ VTE, particularly in cancer, leads to prolonged and more frequent hospitalizations, and has a substantial impact on quality of life. ${ }^{6,7}$ Complications of VTE, such as recurrence, post-thrombotic syndrome and treatment-related bleeding, occur more frequently in cancer patients, ${ }^{6,8,9}$ and the risk of death is higher in cancer patients with than without VTE. .10,11 $^{11}$

Family and twin studies suggest that VTE is highly heritable, and likely results from an interplay between inherited and environmental factors. ${ }^{12,13}$ Fibrinogen, the precursor of fibrin, is an essential component in the final stage of the coagulation cascade. The fibrinogen molecule has three subunits called $A \alpha, B \beta$ and $\gamma$, which occur in pairs for a total number of six subunits. The $\gamma$ chain, transcribed from the
Ferrata Storti Foundation
Haematologica 2020

Volume 105(7):1963-1968

\section{Correspondence:}

BENEDIKTE PAULSEN

benedikte.paulsen@uit.no

Received: April 11, 2019.

Accepted: October 3, 2019.

Pre-published: October 3, 2019.

doi:10.3324/haematol.2019.224279

Check the online version for the most updated information on this article, online supplements, and information on authorship \& disclosures: www.haematologica.org/content/105/7/1963

(C)2020 Ferrata Storti Foundation

Material published in Haematologica is covered by copyright. All rights are reserved to the Ferrata Storti Foundation. Use of published material is allowed under the following terms and conditions:

https://creativecommons.org/licenses/by-nc/4.0/legalcode. Copies of published material are allowed for personal or internal use. Sharing published material for non-commercial purposes is subject to the following conditions:

https://creativecommons.org/licenses/by-nc/4.0/legalcode, sect. 3. Reproducing and sharing published material for commercial purposes is not allowed without permission in writing from the publisher. 
fibrinogen gamma gene (FGG) located on chromosome 4, has two isoforms, $\gamma \mathrm{A}$ and $\gamma^{\prime}$. In the Leiden Thrombophilia Study, the FGG rs2066865 single nucleotide polymorphism (SNP) was first proposed as a risk factor for VTE by reducing fibrinogen ' $\gamma$ ' levels. ${ }^{14}$ Several later genotyping ${ }^{15,16}$ and genome-wide association studies (GWAS) 17,18 confirmed an association between rs2066865 and VTE risk, whereas two cohort studies found no significant association. ${ }^{19,20}$ In a recent metaanalysis including seven studies, rs2066865 was associated with an increased risk of VTE (OR 1.61, 95\% CI: 1.341.93). ${ }^{21}$

The majority of the genetic studies have excluded individuals with cancer-related thrombosis. However, as prothrombotic genotypes are fixed, and not influenced by disease, interventions and complications, they may be attractive candidates as biomarkers of VTE risk in cancer patients. Recent studies have suggested that interactions between cancer and other prothrombotic genotypes (factor $\mathrm{V}$ variants rs6025 and rs4524 and prothrombin G20210A) have synergistic effects on the risk of VTE. ${ }^{22-25}$ To the best of our knowledge, no study has investigated the impact of rs2066865 on the risk of VTE in cancer patients. Therefore, we aimed to investigate the joint effect of rs2066865 and active cancer on the absolute and relative risks of VTE in a population-based case-cohort.

\section{Methods}

\section{Study population}

The Tromsø Study is a single-center population-based cohort, following residents of the municipality of Tromsø, Norway, with repeated health surveys. The case-cohort was derived from the fourth survey (Tromsø 4), which included 27,158 participants aged 25-97 years. A detailed cohort profile of the Tromsø study has been published previously. ${ }^{26}$ The study was approved by the Regional Committee for Medical and Health Research Ethics in Northern Norway, and all participants provided informed written consent to participation. From enrolment in Tromsø 4 (1994/95), subjects were followed until December 31, 2012. Detailed information regarding identification and validation of VTE-events are described in the Online Supplementary Material and Methods.

In total, 710 participants developed VTE during follow-up. Of these, 26 did not have blood samples available or of sufficient quality for DNA analyses. The remaining 684 subjects were included as the cases in our study. A subcohort $(n=3,931)$ was composed by randomly sampling individuals from Tromsø 4 weighted for the age distribution of the cases in 5-year age-groups. Due to the nature of the case-cohort design, where each participant has the same probability of sampling, 72 of the cases were also in the subcohort. Subjects with a history of cancer prior to inclusion $(\mathrm{n}=232)$ and subjects with missing information on rs2066865 ( $\mathrm{n}=9$ ) were excluded from the analysis. The final casecohort consisted of 4,374 subjects, with 640 cases and 3,734 in the subcohort. A flow chart of the case-cohort is displayed in Figure 1.

\section{Baseline measurements and genotyping}

Baseline measurements and genotyping methods are described in the Online Supplementary Materials and Methods.

\section{Cancer exposure}

Cancer assessment is described in the Online Supplementary Materials and Methods. Previous studies have shown a strong temporal relation between cancer diagnosis and incident VTE, and up to $50 \%$ of cancer-related VTE events presents within a 2.5 -year interval (from six months preceding the cancer diagnosis until 2 years following the cancer diagnosis). ${ }^{27,28}$ Therefore, a VTE was defined as related to active cancer if it occurred within this time period.

Subjects who survived the active cancer period without a VTE were censored at the end of the active cancer period (i.e. 2 years after cancer was diagnosed). The censoring was performed because information regarding remission and relapse of cancer was unavailable, and extension of the observation period of cancer could result in the dilution of the estimates due to inclusion of VTE cases not necessarily caused by cancer. This approach resulted in censoring of 14 VTE cases that occurred after the active cancer period. Thus, 626 VTE cases were included in the final analyses.

\section{Statistical analysis}

Statistical analyses were performed using STATA version 15.0 (Stata Corporation LP, College Station, TX, USA). Cox proportional hazards regression models were used to obtain age- and sexadjusted HR with $95 \%$ CI for VTE across categories of cancer status (no cancer/active cancer) and FGG risk alleles. Cancer was assessed as a time-dependent covariate in the model. Subjects who developed cancer contributed person-time as unexposed from the inclusion date until six months prior a cancer diagnosis, and thereafter contributed person-time in the active cancer group as exposed. Absolute incidence rates (IR) were calculated based on person-time from the original cohort $(n=27,128)$. To calculate joint effects conferred by active cancer and FGG risk alleles, subjects with no cancer and no risk alleles were used as the reference group in the Cox model. Based on the total active cancer person-time at risk derived from the source cohort, 1-Kaplan-Meier curves were used to estimate the cumulative incidence of VTE in subjects with active cancer according to the presence of FGG risk alleles. Methods for assessing synergism between FGG and active cancer on the risk of VTE are described in detail in the Online Supplementary Materials and Methods.

\section{Results}

The mean follow-up of the case-cohort was 12.6 years. In total, 854 subjects had active cancer, of which 167 experienced an incident VTE. The baseline characteristics of

Table 1. Baseline characteristics in the entire case-cohort and in the active cancer group.

\begin{tabular}{lcc} 
& Entire case-cohort & Active cancer \\
Subjects $(\mathrm{n})$ & 4374 & 854 \\
Age (years) & $58 \pm 13$ & $62 \pm 10$ \\
\hline Sex (males) & $47.0(2,048)$ & $53.0(456)$ \\
BMI $\left(\mathrm{kg} / \mathrm{m}^{2}\right)$ & $26.0 \pm 4$ & $26.0 \pm 4$ \\
\hline Daily smoking & $34.5(1,464)$ & $43.5(364)$ \\
WBC count $\left(10^{9} / \mathrm{L}\right)$ & $7.1 \pm 1.8$ & $7.2 \pm 1.8$ \\
\hline Platelet count $\left(10^{9} / \mathrm{L}\right)$ & $251 \pm 60$ & $250 \pm 58$ \\
rs2066865* & 0.26 & 0.26 \\
1 risk allele & 1,723 & 334 \\
2 risk alleles & 289 & 51 \\
\hline
\end{tabular}

Values are numbers or percentages with numbers in parenthesis or means \pm standard deviation (SD). Active cancer:period from six months before a cancer diagnosis until two years after; BMI: body mass index; Daily smoking indicates smoking at the time of enrollment;WBC: white blood cell; * : allele frequency. 
the entire case-cohort and in those with active cancer during follow-up are presented in Table 1. Subjects who developed active cancer were slightly older $(61 \pm 10$ years vs. $58 \pm 13$ years) and reported a higher frequency of daily smoking $(46 \%$ vs. $35 \%)$ compared to the entire casecohort. The minor allele frequency of $r s 2066865$ was 0.26 , which is comparable to reference populations. ${ }^{14,29}$ The homozygous variant of the FGG was present in 289 $(6.6 \%)$ subjects, the heterozygous variant in 1,723 (39.4\%) subjects, while 2,362 (54.0\%) subjects were non-carriers of the FGG variant. The allele frequency was essentially similar in subjects who developed cancer. Expected versus observed proportions of hetero- and homozygous individuals in the subcohort according to the Hardy-Weinberg equilibrium are presented in Online Supplementary Table S1.

The clinical characteristics of the VTE events stratified by the presence of active cancer are shown in Table 2 . Compared to the non-cancer-related VTE, cancer-related VTE were more often a DVT $(59.2 \%$ vs. $55.5 \%)$ than a PE ( $40.7 \%$ vs. $44.4 \%)$. The prevalence of provoking factors such as acute medical conditions, immobilization and surgery were essentially similar between the two groups, as were the total proportion of VTE with one or more concurrent provoking factors ( $44.3 \%$ vs. $44.7 \%)$ ). Non-cancer related VTE were more likely to be associated with traumas $(9.6 \%$ vs. $2.4 \%)$ while other provoking factors (i.e. venous catheters) were more frequent in cancer-related VTE $(8.4 \%$ vs. $3.7 \%)$.

In participants without cancer, the IR of VTE increased from 1.2 (95\% CI: 1.1-1.4) per 1,000 people/year among non-carriers of FGG rs2066865 to 2.0 (95\% CI: 1.5-2.7) per 1,000 people/year among those with two risk alleles. Accordingly, the risk of VTE was $70 \%$ (HR 1.7, 95\% CI: 1.2-2.3) higher in those with two risk alleles at FGG compared to non-carriers (Table 3 ). In subjects with active cancer, the risk was 12-fold higher (HR 11.9, 95\% CI: 9.3$15.2)$ in those with no $F G G$ risk alleles, and 22-fold higher (HR 22.2, 95\% CI: 12.9-38.1) in those with two FGG risk alleles, compared to cancer-free subject without risk alleles. Cancer patients with two risk alleles at FGG had a two-fold higher (HR 2.0, 95\% CI 1.1-3.6) risk of VTE compared to cancer patients without risk alleles. In sub-analyses, the effect of active cancer and homozygosity at FGG yielded higher risk estimates for PE (HR 2.9, 95\% CI: 1.36.6) than for DVT (HR 1.6, 95\% CI: 0.7-3.5).

The cumulative incidence of VTE during the active can- cer period is shown in Figure 2. The cumulative incidence of VTE increased particularly during the first six months following a cancer diagnosis, where we found a substantially steeper incline in the incidence curve for subjects with two risk alleles at FGG rs2066865. The cumulative incidence of VTE among homozygous carriers was 5.0\% (95\% CI: $2.4-9.6$ ), $6.4 \%$ (95\% CI: $3.5-11.6)$, and $8.0 \%$ (95\% CI: 4.6-13.9) at three months, six months and 24 months after cancer diagnosis, respectively. The corresponding figures for cancer patients who were non-carriers were $2.1 \%$ (95\% CI: $1.5-3.0), 3.1 \%$ (95\% CI: $2.3-4.7$ ), and $4.8 \%$ (95\% CI: 3.8-6.2), respectively.

A supra-additive effect on the risk of VTE was observed for the combination of homozygosity at the $F G G$ variant and active cancer (Table 4). The Relative excess risk by

Table 2. Characteristics of subjects with cancer-related and non-cancer-related first venous thromboembolism.

\begin{tabular}{lcc} 
& \multicolumn{2}{c}{ Cancer-related VIE } \\
& Yes (167) & No (459) \\
Age at VTE diagnosis (years) & $69 \pm 11$ & $68 \pm 14$ \\
Sex (Males) & $44.9(75)$ & $47.3(217)$ \\
\hline VTE type & & \\
Deep vein thrombosis & $59.2(99)$ & $55.5(255)$ \\
Proximal upper limb & $5.1(5)$ & $2.0(5)$ \\
Distal upper limb & $1.0(1)$ & $0(0)$ \\
Proximal lower limb & $62.6(62)$ & $65.9(168)$ \\
Distal lower limb & $12.1(12)$ & $28.2(72)$ \\
Other localizations & $19.1(19)$ & $3.9(10)$ \\
Pulmonary embolism & $40.7(68)$ & $44.4(204)$ \\
Unprovoked event & NA & $54.9(252)$ \\
Provoking factors $^{2}$ & \\
Surgery & & \\
Trauma $^{\mathrm{a}}$ & $12.6(21)$ & $15.3(70)$ \\
Acute medical condition $^{\mathrm{b}}$ & $2.4(4)$ & $9.6(44)$ \\
Immobilization $^{\mathrm{c}}$ & $15.0(25)$ & $14.2(65)$ \\
Other provoking factor $^{\mathrm{d}}$ & $20.4(34)$ & $20.0(92)$ \\
Total provoked $^{\mathrm{a}}$ & $8.4(14)$ & $3.7(17)$ \\
\hline
\end{tabular}

Values are numbers or percentages with numbers in parenthesis or means \pm standard deviation (SD); VTE: venous thromboembolism; NA: not applicable; "within eight weeks before the VTE-event; ${ }^{b}$ myocardial infarction, ischemic stroke of major infectious disease; cbedrest $>3$ days, wheelchair, long haul travel $>4$ hours in the past 14 days; ${ }^{\circledR}$ presence of other provoking factors noted by the physician (e.g. intravenous catheters); ${ }^{\mathrm{e}}$ one or more provoking factor above

Table 3. Age and sex adjusted hazard ratios for venous thromboembolism according to categories of fibrinogen gamma (FGG) risk alleles and cancer status.

\begin{tabular}{|c|c|c|c|c|c|c|c|c|c|c|}
\hline & \multirow[b]{2}{*}{ Risk Alleles } & \multicolumn{3}{|c|}{ VE } & \multicolumn{3}{|c|}{ PE } & \multicolumn{3}{|c|}{ DVI } \\
\hline & & Events & $\begin{array}{c}\mathrm{HR} \\
(95 \% \mathrm{Cl})\end{array}$ & $\begin{array}{c}\text { HR } \\
(95 \% \text { Cl) }\end{array}$ & Events & $\begin{array}{c}\text { HR } \\
(95 \% \text { CI) }\end{array}$ & $\begin{array}{c}\text { HR } \\
(95 \% \text { Cl) }\end{array}$ & Events & $\begin{array}{c}\mathrm{HR} \\
(95 \% \mathrm{CI})\end{array}$ & $\begin{array}{c}\mathrm{HR} \\
(95 \% \mathrm{CI})\end{array}$ \\
\hline \multirow{3}{*}{ No cancer } & 0 & 242 & Ref. & - & 112 & Ref. & - & 130 & Ref. & - \\
\hline & 1 & 170 & $1.0(0.8-1.2)$ & - & 70 & $0.9(0.6-1.2)$ & - & 100 & $1.1(0.8-1.4)$ & - \\
\hline & 2 & 47 & $1.7(1.2-2.3)$ & - & 22 & $1.7(1.1-2.7)$ & - & 25 & $1.6(1.1-2.5)$ & - \\
\hline \multirow{3}{*}{ Active cancer } & 0 & 89 & $11.9(9.3-15.2)$ & Ref. & 32 & $8.3(5.6-12.5)$ & Ref. & 57 & $15.3(11.2-21.1)$ & Ref. \\
\hline & 1 & 64 & $12.2(9.2-16.1)$ & $1.1(0.8-1.5)$ & 29 & $10.6(7.1-16.3)$ & $1.3(0.8-2.2)$ & 35 & $13.4(9.2-19.6)$ & $1.0(0.6-1.5)$ \\
\hline & 2 & 14 & $22.2(12.9-38.1)$ & $2.0(1.1-3.6)$ & 7 & $22.8(10.6-49.1)$ & $2.9(1.3-6.6)$ & 7 & $21.6(10.0-46.4)$ & $1.6(0.7-3.5)$ \\
\hline
\end{tabular}

Active cancer: period from six months before a cancer diagnosis until two years after; CI: confidence interval; DVT: deep vein thrombosis; HR: hazard ratio; PE: pulmonary embolism;VTE: venous thromboembolism. 
interaction (RERI) was 9.61 (95\% CI: -2.38-21.61) and the Rothmans synergy index (RSI) was 1.81 (95\% CI: 1.023.21). The proportion attributable to interaction (AP) was 0.43 (95\% CI: 0.11-0.74). In sub-group analysis, the estimates of biological interaction were stronger for $\mathrm{PE}$ (RSI=2.37, 95\% CI: 1.05-5.39) than for DVT (RSI=1.46, 95\% CI: 0.65-3.27).

\section{Discussion}

In the present study, we aimed to investigate the joint effect of the rs2066865 SNP at FGG and active cancer on the risk of VTE in a case-cohort recruited from the general population. Homozygosity at rs2066865, occurring in $6.6 \%$ of the study population, was associated with an increased risk of VTE. The combination of an rs 2066865 homozygous risk genotype and active cancer showed a synergistic effect on VTE risk (on an additive scale). The effect was particularly strong for PE. The cumulative incidence of VTE increased substantially during the first six months following a cancer diagnosis, especially among patients with two risk alleles at FGG rs2066865. Our findings suggest that homozygosity at FGG rs2066865 may aid to differentiate patients at high and low risk of cancerrelated VTE.

Several observational studies have reported an association between homozygous genotype of rs2066865 and increased risk of VTE in Caucasians. ${ }^{14-16,21}$ In a recent metaanalysis including seven observational studies, the odds ratio of VTE was 1.61 for homozygosity at rs2066865. ${ }^{21}$ Accordingly, in cancer-free subjects, we found that those with two rs2066865 risk alleles had a 1.7-fold higher VTE risk than those with 0 risk alleles. The risk estimates for DVT and PE were essentially similar in cancer-free subjects.

Even though the role of prothrombotic genotypes in cancer-related VTE have been scarcely studied, previous studies have found that some prothrombotic genotypes (e.g. factor V Leiden and prothrombin G20210A) are associated with increased risk of cancer-related VTE. $22,23,30,31$ Further, the combined effect of cancer and factor $V$ variants (factor V Leiden and rs4524) exceeded the sum of the individual effects, implicating a biological interaction on VTE risk. ${ }^{22,24}$ Accordingly, we found that the combination of FGG and active cancer yielded a synergistic effect on VTE risk.

In cancer patients, the cumulative incidence curve of VTE was substantially steeper in individuals homozygous for FGG during the first six months following the cancer diagnosis. According to the thrombosis potential model, ${ }^{32}$ several risk factors need to be present concurrently to exceed the thrombosis potential and facilitate development of a VTE. In the period following a cancer diagnosis, treatment with surgery and/or chemotherapy is typically initiated, and treatment-related complications such as acute infection and immobilization frequently occur. Thus, the accumulation of several treatment-related risk factors, which adds to the background risk in patients with cancer and risk alleles at FGG, may partly explain the substantial increase in VTE incidence the first half year following a cancer diagnosis.

In contrast to cancer-free subjects, we found that the effect of rs2066865 was stronger for PE than for DVT in cancer patients. This suggests that the FGG variant may

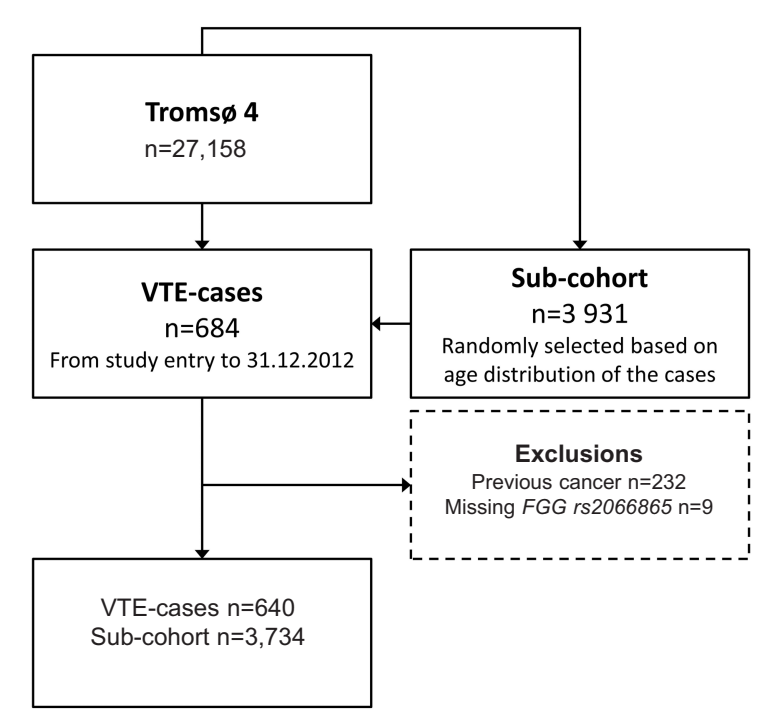

Figure 1. Flow chart for the case-cohort.

play a more essential role in the pathogenesis of PE than DVT in cancer patients. The underlying mechanism(s) for the latter observation is unknown, but may imply that rs2066865 is associated with fragile thrombi, which are prone to embolization and manifest clinically as PE rather than DVT in cancer patients.

The mechanism by which the rs2066865 affects susceptibility to VTE is not fully elucidated. However, the current hypothesis is that it acts through a phenotype with altered fibrinogen composition and formation. The rs2066865 SNP tags the FGG-H2 haplotype. Previous studies have shown that homozygous carriers of the FGG$\mathrm{H} 2$ haplotype had lower levels of $\gamma^{\prime}$ fibrinogen and $\gamma^{\prime}$ fibrinogen/total fibrinogen concentration ${ }^{14}$ without alterations in the total fibrinogen level. ${ }^{33}$ The suggested mechanism is that the FGG variant favors formation of the abundant $\gamma$-chain isoform $(\gamma \mathrm{A})$ above the minor $\gamma$-chain $\left(\gamma^{\prime}\right)$ through alternative splicing of the mRNA of the FGGgene. ${ }^{14,33}$ Fibrinogen $\gamma^{\prime}$ exhibits an inhibitory activity towards thrombin, due to a high affinity binding site on the $\gamma^{\prime}$ chain for thrombin exosite $\mathrm{II}^{34}$ which inhibits thrombin-mediated activation of factor VIII, ${ }^{35}$ factor $\mathrm{V}^{36}$ and platelets. ${ }^{37}$ Moreover, fibrinogen $\gamma^{\prime}$ has been shown to increase the activated protein C (APC) sensitivity. ${ }^{38}$ However, studies on the association between low plasma levels of fibrinogen $\gamma^{\prime}$ and VTE risk have shown somewhat inconsistent results. ${ }^{14,20}$

Current anticoagulant prophylaxis regimens efficiently prevent first VTE in cancer patients, but at the expense of a substantial risk of major and life-threatening bleedings. ${ }^{39}$ Therefore, current international guidelines do not recommend prophylactic anticoagulation to all ambulatory cancer patients. ${ }^{40,41}$ Thus, it is vital to recognize patients that are at high risk of cancer associated VTE, in order to identify those who would benefit most from thromboprophylaxis. Prothrombotic genotypes are attractive biomarker candidates, which could be used to distinguish between high and low risk of VTE in cancer patients, since they are fixed and not affected by the clinical status or treatmentrelated factors. In the present study, $6.4 \%$ of cancer 


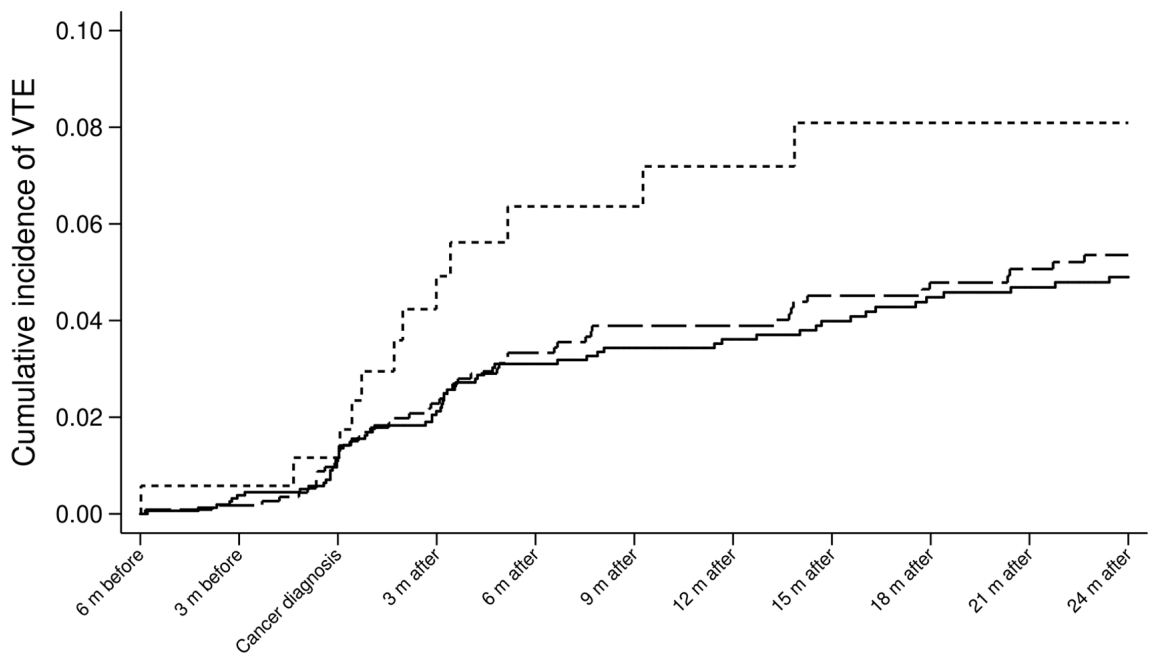

Figure 2. Cumulative incidence of venous thromboembolism in the presence of FGG rs2066865 risk alleles during the active cancer period. VTE:

Active cancer period (3 months intervals) venous thromboembolism; m: months.

Table 4. Measures of interaction between the homozygous fibrinogen gamma (FGG) variant and active cancer on venous thromboembolism.

\begin{tabular}{lccc} 
& $\begin{array}{c}\text { Rothmans synergy index (RSI) } \\
(95 \% \text { CI) }\end{array}$ & $\begin{array}{c}\text { Relative excess risk by interaction (RERI) } \\
(95 \% \text { CI) }\end{array}$ & $\begin{array}{c}\text { Proportion due to interaction (AP) } \\
(95 \% \text { CI) }\end{array}$ \\
FGG rs2066865 & & $0.43(0.11-0.74)$ \\
VTE & $1.81(1.02-3.21)$ & $9.6(-2.4-21.6)$ & $0.56(0.21-0.90)$ \\
PE & $2.37(1.05-5.39)$ & $13.4(-4.8-31.7)$ & $0.30(-0.24-0.83)$ \\
\hline DVT & $1.46(0.65-3.27)$ & $6.3(-9.6-22.1)$ & \\
\hline
\end{tabular}

Rothmans synergy index (RSI) $>1$ indicates a positive interaction or more than additivity; Relative excess risk by interaction (RERI) $>0$ indicates a positive interaction or more than additivity; Proportion due to interaction (AP) $>0$ indicates a positive interaction or more than additivity.VTE: venous thromboembolism; PE: pulmonary embolism; DVT: deep vein thrombosis; CI: confidence interval.

patients with two risk alleles at FGG rs2066865 developed VTE during the first six months after cancer diagnosis compared to $3.1 \%$ of cancer patients without risk alleles. Our findings suggest that FGG may be an attractive gene candidate to pursue in future research on prediction models of VTE risk in cancer patients. We and others have previously reported similar discriminative power of two variants in the F5 gene (rs6025 and rs4524), ${ }^{23,24}$ and a genetic model including nine SNP reported promising predictive capacity on VTE risk in breast cancer. ${ }^{42}$ Recently, a new risk prediction model for cancer-related VTE, including clinical characteristics and genetic variants, reported a strong predictive capacity with an area under the curve (AUC) of 0.73 and performed better that the Khorana score (AUC 0.58). ${ }^{43}$

The main strengths of present study are the prospective design, high participation rate and long-term follow-up, making it possible to capture a large quantity of both incident cancer- and VTE-events in the study population. Since all participants live within a single hospital catchment area, the probability of missing outcomes is low.
Moreover, both incident VTE-events and cancer diagnoses were systematically validated and objectively confirmed. The study was limited by the lack of statistical power in sub-group analysis (i.e. DVT/PE), illustrated by wide CI for our risk estimates. In addition, we did not have access to information on treatment regimens or medical complications among cancer patients. Although there is no reason to believe that the type or intensity of treatment would be influenced by the genetic makeup, such data could have provided further insights into the possible interplay between genes and treatment-related risk factors.

In conclusion, we found that homozygosity at $F G G$ rs 2066865 was associated with an increased risk of VTE, and yielded a synergistic effect on the VTE risk in combination with active cancer, particularly on the risk of $\mathrm{PE}$.

\section{Funding}

The K.G. Jebsen Thrombosis Research and Expertise Centre is supported by an independent grant from Stiftelsen Kristian Gerhard Jebsen.

\section{References}

1. Heit JA. Venous thromboembolism: disease burden, outcomes and risk factors. J Thromb Haemost. 2005;3(8):1611-1617.

2. White RH. The epidemiology of venous thromboembolism. Circulation. 2003;107(23 Suppl 1):14-8.

3. Naess IA, Christiansen SC, Romundstad P,
Cannegieter SC, Rosendaal FR, Hammerstrom J. Incidence and mortality of venous thrombosis: a population-based study. J Thromb Haemost. 2007;5(4):692699.

4. Spencer FA, Lessard D, Emery C, Reed G, Goldberg RJ. Venous thromboembolism in the outpatient setting. Arch Intern Med. 2007;167(14):1471-1475.
5. Timp JF, Braekkan SK, Versteeg $\mathrm{HH}$ Cannegieter SC. Epidemiology of cancerassociated venous thrombosis. Blood 2013;122(10):1712-1723.

6. Prandoni P, Lensing AW, Piccioli A, et al Recurrent venous thromboembolism and bleeding complications during anticoagulant treatment in patients with cancer and venous thrombosis. Blood. 2002;100(10): 
B. Paulsen et al.

3484-3488.

7. Elting LS, Escalante CP, Cooksley C, et al. Outcomes and cost of deep venous throwboss among patients with cancer. Arch Intern Med. 2004;164(15):1653-1661.

8. Lee KW, Lip GY. Effects of lifestyle on hemostasis, fibrinolysis, and platelet reactivity: a systematic review. Arch Intern Med. 2003;163(19):2368-2392.

9. Noble S, Parsi J. Epidemiology and pathophysiology of cancer-associated thrombosis. Br J Cancer. 2010;102 Suppl 1:S2-9.

10. Khorana AA, Francis CW, Culakova E, Kuderer NM, Lyman GH. Frequency, risk factors, and trends for venous thromboemholism among hospitalized cancer patients. Cancer. 2007;110(10):2339-2346.

11. Sorensen HT, Mellemkjaer L, Olsen JH Baron JA. Prognosis of cancers associated with venous thromboembolism. N Angl J Med. 2000;343(25):1846-1850.

12. Larsen TB, Sorensen HT, Skytthe A, Johnsen SP, Vaupel JW, Christensen K. Major genetic susceptibility for venous thromboembolism in men: a study of Danish twins. Epidemiology. 2003;14(3):328-332.

13. Soto JC, Almasy L, Barrel M, et al. Genetic susceptibility to thrombosis and its relationship to physiological risk factors: the GAIT study. Genetic Analysis of Idiopathic Thrombophilia. Am J Hum Genet. 2000;67(6):1452-1459.

14. Nite de Willie S, de Visser MC, HousingDuistermaat JJ, Rosendaal FR, Voc HL, Bertina RM. Genetic variation in the fibrinogen gamma gene increases the risk for deep venous thrombosis by reducing alasma fibrinogen gamma' levels. Blood. 2005;106(13):4176-4183.

15. Uitte de Willie S, Pyle ME, Vo HL, et al. Fibrinogen gamma gene 3 '-end polymorphisms and risk of venous thromboemholism in the African-American and Caucasian population. Thromb Haemost. 2009;101(6):1078-1084.

16. Grunbacher G, Weger W, Marx-Neuhold E, et al. The fibrinogen gamma (FGG) 10034C $>\mathrm{T}$ polymorphism is associated with venous thrombosis. Thromb Res. 2007;121(1):33-36.

17. Germain M, Shut N, Greliche N, et al. Genetics of venous thrombosis: insights from a new genome wide association study. PLo One. 2011;6(9):e25581.

18. Germain M, Chasman DI, de Wan H, et al. Meta-analysis of 65,734 individuals identifie TSPAN15 and SLC44A2 as two susceptibility loci for venous thromboembolism. Am J Hum Genet. 2015;96(4):532-542.

19. El-Galaly TC, Severinsen MT, Overvad K, et al. Single nucleotide polymorphisms and the risk of venous thrombosis: results from a Danish case-cohort study. Br J Haematol. 2013;160(6):838-841.

20. Folsom AR, Tang W, George KM, et al. Prospective study of gamma' fibrinogen and incident venous thromboembolism: The Longitudinal Investigation of Thromboembolism Etiology (LITE). Thromb Res. 2016;139:44-49.

21. Jiang J, Lu K, Zou J, et al. Associations between polymorphisms in coagulationrelated genes and venous thromboembolism: A meta-analysis with trial sequintrial analysis. Medicine (Baltimore). 2017; 96(13):e6537.

22. Blow JW, Doggen CJ, Osanto S, Rosendaal FR. Malignancies, prothrombotic mutatrons, and the risk of venous thrombosis. JAMA. 2005;293(6):715-722.

23. Pabinger I, Av C, Dunker D, et al. Factor V Leiden mutation increases the risk for venous thromboembolism in cancer patients - results from the Vienna Cancer And Thrombosis Study (CATS). J Thromb Haemost. 2015;13(1):17-22.

24. Gran OV, Smith EN, Braekkan SK, et al. Joint effects of cancer and variants in the Factor 5 gene on the risk of venous throwboembolism. Haematological. 2016; 101(9):1046-1053.

25. Gran OV, Braekkan SK, Hansen JB. Prothrombotic genotypes and risk of venous thromboembolism in cancer. Thromb Res. 2018;164 Suppl 1:S12-S18.

26. Jacobsen BK, Egger AE, Mathiesen EB, Wilsgaard T, Njolstad I. Cohort profile: the Tromso Study. Int J Epidemiol. 2012; 41(4):961-967.

27. Blix K, Severinsen MT, Braekkan S, et al. Cancer-related venous thromboembolism in the general population: results from the Scandinavian Thrombosis and Cancer (STAC) study. J Thromb Haemost. 2015;13:(Suppl 2):549-549.

28. White RH, Chew HK, Zhou $\mathrm{H}$, et al. Incidence of venous thromboembolism in the year before the diagnosis of cancer in 528,693 adults. Arch Intern Med. 2005;165(15):1782-1787.

29. Morange PE, Tregouet DA. Lessons from genome-wide association studies in venous thrombosis. J Thromb Haemost. 2011;9(Suppl 1):258-264.

30. Ramacciotti E, Wolosker N, Puech-Leao P, et al. Prevalence of factor V Leiden, FII G20210A, FXIII Va134Leu and MTHFR C677T polymorphisms in cancer patients with and without venous thrombosis. Thromb Res. 2003;109(4):171-174.

31. Mandala M, Curigliano G, Bucciarelli P, et al. Factor V Leiden and G20210A prothrombin mutation and the risk of subclavan vein thrombosis in patients with breast cancer and a central venous catheter.
Ann Oncol. 2004;15(4):590-593.

32. Rosendaal FR. Venous thrombosis: a multicausal disease. Lancet. 1999; 353(9159):1167-1173.

33. Willie SU, Rietveld IM, De Wiser $\mathrm{MCH}$, Voc HL, Bertina RM. Polymorphism $10034 \mathrm{C}>\mathrm{T}$ is located in a region regulating polyadenylation of FGG transcripts and influences the fibrinogen gamma'/gamma A mRA ratio. J Thromb Haemost. 2007;5(6):1243-1249.

34. Farrell DH. gamma' Fibrinogen as a novel marker of thrombotic disease. Chin Chem Lab Med. 2012;50(11):1903-1909.

35. Lovely RS, Boshkov LK, Marzec UM, Hanson SR, Farrell DH. Fibrinogen gamma' chain carboxy terminal peptide selectively inhibits the intrinsic coagulation pathway. Br J Haematol. 2007;139(3):494-503.

36. Omarova F, Nite De Willie $S$, Ariens RA, Posing J, Bertina RM, Castoldi E. Inhibition of thrombin-mediated factor $\mathrm{V}$ activation contributes to the anticoagulant activity of fibrinogen gamma'. J Thromb Haemost. 2013;11(9):1669-1678.

37. Lovely RS, Rein CM, White TC, et al. gammaA/gamma' fibrinogen inhibits thrombin-induced platelet aggregation. Thromb Haemost. 2008;100(5):837-846.

38. Omarova F, Uitte de Willie S, Simioni P, et al. Fibrinogen gamma' increases the sensetivity to activated protein $\mathrm{C}$ in normal and factor V Leiden plasma. Blood. 2014; 124(9):1531-1538.

39. Di Nisio M, Porreca E, Ferrante N, Otten HM, Cuccurullo F, Rutjes AW. Primary prophylaxis for venous thromboembolism in ambulatory cancer patients receiving chemotherapy. Cochran Database Syst Rev. 2012;(2):CD008500.

40. Kahn SR, Him W, Dunn AS, et al. Prevention of VTE in nonsurgical patients: Antithrombotic Therapy and Prevention of Thrombosis, 9th ed: American College of Chest Physicians Evidence-Based Clinical Practice Guidelines. Chest. 2012;141(2 Suppl):e195S-e226S.

41. Lyman GH, Bohlke K, Khorana AA, et al. Venous thromboembolism prophylaxis and treatment in patients with cancer: american society of clinical oncology clinical practice guideline update 2014. J Chin Oncol. 2015; 33(6):654-656.

42. Brand JS, Hedayati E, Humphreys K, et al. Chemotherapy, genetic susceptibility, and risk of venous thromboembolism in breast cancer patients. Chin Cancer Res. 2016;22(21):5249-5255.

43. Munoz Martin AJ, Ortega I, Font C, et al. Multivariable clinical-genetic risk model for predicting venous thromboembolic events in patients with cancer. Br J Cancer. 2018; 118(8):1056-1061.

1968

haematological | 2020; 105(7) 